\title{
Nonlinear Adaptive Control for Bioreactors with Unknown Kinetics *
}

\author{
Ludovic Mailleret $^{\mathrm{a}}$, Olivier Bernard ${ }^{\mathrm{a}}$, Jean-Philippe Steyer ${ }^{\mathrm{b}}$ \\ ${ }^{a}$ COMORE, INRIA, BP93, 06902 Sophia-Antipolis cedex, France \\ ${ }^{\mathrm{b}}$ LBE, INRA, Avenue des étangs, 11100 Narbonne, France
}

\begin{abstract}
We consider a control problem for a single bioreaction occuring in a continuous and well mixed bioreactor, assuming that the bioreaction's kinetics is not represented by a validated model. We develop a nonlinear controller and prove the global asymptotic stability of the closed loop system towards the equilibrium corresponding to the set point. Since this control law needs the knowledge of some parameters, we derive an adaptive version of the nonlinear controller and prove again the global asymptotic stability of the closed loop system. Finally, we show the relevance of our approach on a real life wastewater treatment plant.
\end{abstract}

Key words: Nonlinear adaptive control, continuous bioprocesses, unknown kinetics, wastewater treatment.

\section{Introduction}

Biological processes have become widely used in the industry for the last decades, with different purposes: either to produce some chemical compounds synthesized by a microorganism (alcoholic fermentation...), to cultivate a biomass for its utilization (baker's yeast...) or extraction of its metabolites (carotene from plankton...), or to degrade a pollutant (wastewater treatment...). Therefore, bioreactors require advanced regulation procedures to ensure the bioprocesses' performances and efficiency.

However, the control of bioreactors is a delicate problem since most of the time the available biological models are only rough approximations. Indeed, biological systems are known to be highly variable and difficult to measure so that no reliable biological law is available. A way to circumvent this difficulty is the mass-balance based modelling (Bastin \& Dochain, 1990): the biological lacks of knowledge are located in dedicated terms, namely the bioreaction's kinetics.

In this paper we focus on continuous bioprocesses; some different approaches for their control can be found in the

\footnotetext{
* This paper was not presented at any IFAC meeting. Corresponding author: L. Mailleret. Fax +33492387858.

Email addresses: ludovic.mailleret@inria.fr

(Ludovic Mailleret), olivier.bernard@inria.fr (Olivier Bernard), steyer@ensam.inra.fr (Jean-Philippe Steyer).
}

literature. The three main trends are: local approaches, global approaches based on full model knowledge and global approaches taking into account some model uncertainties. Local approaches (Heinzle, Dunn \& Ryhiner, 1993) use the linearized model around the desired operating point together with linear systems control's results. Global approaches are mainly linearizing controllers (Perrier \& Dochain, 1993; Bastin \& Van Impe, 1995; Proell \& Karim, 1994), using full model knowledge for exact model linearization. The main drawback of these global approaches is that they use perfect model knowledge. Other global control techniques assuming model uncertainties arise (Rapaport \& Harmand, 2002), using interval observers results (Gouzé, Rapaport \& Hadj-Sadok, 2000). However, a drawback common to most of the aforementioned control strategies is that they often do not explicitly take into account the nonnegativity constraints on the manipulated variables.

We consider in this paper a simple class of bioreactions, often used to describe the growth of a single species of microorganisms (Bailey \& Ollis, 1986). Here, we only assume qualitative hypotheses on the involved biological phenomena: we do not suppose any analytical expression for the kinetic function. Therefore our approach applies to a broad class of microbial species. Despite these modelling uncertainties, we develop a regulation procedure, which guarantees the desired closed loop behavior of the bioprocess: stabilization towards a chosen operating point, even unreachable in open loop. Then, we de- 
rive an adaptive version of this regulation procedure, to take into account parameters uncertainty and/or temporal evolution. Both results are global. Finally, we illustrate the performance of such a controller with both numerical simulation and real data on an efficient but unstable wastewater treatment plant (WWTP), using anaerobic digestion (AD).

\section{General Model Description}

We focus on a simple bioreaction occurring in a Continuous Stirred Tank Reactor (CSTR). We consider a reaction involving microorganisms of a single species $(X)$, growing on a substrate $(S)$ and yielding a product $(P)$. This reaction, which can result from a first approximation of a more complex reaction network, is written:

$k S \stackrel{r(\cdot)}{\longrightarrow} X+h P$

The reaction rate is given by $r($.$) , this notation means$ that we do not specify yet the variables which influence it. The parameters $k$ and $h$ are yield coefficients associated with biomass growth and product synthesis.

\subsection{The model for bioreactions in CSTR}

Concentrations in the liquid phase are supposed to be homogeneous in a CSTR. However, note that a part of the biomass (and/or the product) can be attached (fixed bed bioreactors). A liquid flow passes through the reactor, the inflow feeds the reactor with the substrate $S$ at a concentration $s_{i n}$. The outflow is composed by the same compounds than in the liquid phase of the reactor, substrate $S$, biomass $X$ and product $P$ at concentrations $s$, $x$ and $p$ respectively. According to classical mass-balance based modelling (Bastin \& Dochain, 1990), the state variables $s, x, p$ are solutions of the following system of differential equations:

$\left\{\begin{array}{l}\dot{x}=r(.)-\alpha u x \\ \dot{s}=u\left(s_{i n}-s\right)-k r(.) \\ \dot{p}=h r(.)-\beta u p\end{array}\right.$

where $u$ is the dilution rate (the nonnegative manipulated variable). In the sequel, we will denote $\xi=(x, s, p)$ the state vector. We suppose that only a constant proportion $(\alpha \in(0,1])$ of biomass $X$ is not attached on the support and thus is affected by dilution effects (Bernard, Hadj-Sadok, Dochain, Genovesi \& Steyer, 2001). We define $\beta \in(0,1]$ a coefficient of product non-fixation.

\subsection{The r(.) modelling issue}

The most crucial problem in solving equations (2) is the formulation of a reasonable expression for the kinetic function $r($.$) . As described by Bastin \& Dochain (1990),$ a large number of analytical expressions have been proposed to describe these kinetics. Here, we do not assume any analytical expression for the function $r($.$) , we only$ make the following assumptions based on biological evidence:

Hypothesis $\mathbf{1}$ (H1): We assume that: $a: r($.$) is nonnegative and is at least a function of s$ and $x$ $b: \forall s, x$ positive, $r(s, x, .$.$) is positive$ $c: r(s, x, .$.$) is a \mathcal{C}^{1}$ function

Note that with (H1c), Cauchy conditions for uniqueness of trajectories of (2) are fulfilled (Khalil, 1992). Since most kinetics described in the literature verify these hypotheses (e.g. laws of Monod, Haldane, Contois... see Bastin \& Dochain (1990)), our work is very general. However, in order to design our controller, we need another hypothesis:

Hypothesis 2 (H2): We assume that the quantity: $y_{1}=\lambda r(s, x, .$.$) ( \lambda$ denoting a positive constant) is available online from the plant.

This hypothesis is again very general and almost as applicable as (H1). Real sensors or numerical estimators (Farza, Busawon \& Hammouri, 1998) can indeed be used to obtain online the quantity $y_{1}$. Remark that, for a large part of bioprocesses, the production (or consumption) of gaseous components $\left(\mathrm{O}_{2}, \mathrm{CO}_{2} \ldots\right)$ is monitored and is directly related to the reaction kinetics, therefore to $y_{1}$ (Mailleret, Bernard \& Steyer, 2003). In the sequel, we will suppose that hypotheses (H1) and (H2) hold.

\section{Controllers Design}

Depending on the bioprocess purpose, we want to globally regulate either the substrate concentration $s$, the product concentration $p$ or the biomass concentration $x$. However, in each case, we will see later that the values of $x$ and $p$ obtained at equilibrium, are completely determined by the value of the targeted set point $s^{\star}$ for substrate concentration. Then, in the sequel, we will only focus on the $s$ regulation problem.

We propose in this section an output feedback controller, that achieves the global asymptotic stabilization of a bioprocess, without any knowledge of its kinetics and with respect to the non-negativity constraint of the input. However, this static controller requires accurate knowledge of the parameters $k, s_{i n}$ and $\lambda$ to achieve asymptotic regulation without error. We propose thus an adaptive control law performing exact regulation towards the set point despite parameter uncertainty. 


\subsection{Simple nonlinear controller design}

Let us denote $s^{\star} \in\left(0, s_{i n}\right)$ the desired set point for substrate concentration. We compute the corresponding positive equilibrium values of the two last state variables: $x^{\star}=\frac{1}{\alpha k}\left(s_{i n}-s^{\star}\right)$ and $p^{\star}=\frac{h}{\beta k}\left(s_{i n}-s^{\star}\right)$.

Proposition 1: Under assumptions (H1) and (H2), the nonlinear feedback control law:

$u()=.\frac{k}{\lambda\left(s_{i n}-s^{\star}\right)} y_{1}=\frac{k}{\left(s_{i n}-s^{\star}\right)} r(s, x, .$.

globally stabilizes system (2) towards the positive set point $\xi^{\star}=\left(x^{\star}, s^{\star}, p^{\star}\right)$.

Proof: We have the following closed loop system:

$$
\left\{\begin{array}{l}
\dot{x}=\alpha u(.)\left(x^{\star}-x\right) \\
\dot{s}=u(.)\left(s^{\star}-s\right) \\
\dot{p}=\beta u(.)\left(p^{\star}-p\right)
\end{array}\right.
$$

From (H1a), it is straightforward that the non-negative orthant of the state space is positively invariant by system (4). Thus, for any positive initial state conditions (that are assumed to be positive throughout the paper), the control variable $u($.$) is such that: u() \geq$.0 . Integrating system (4) and since $u() \geq$.0 , we show that $\forall t \geq 0$ :

$$
\left\{\begin{array}{l}
\max \left(x^{\star}, x(0)\right) \geq x(t) \geq \min \left(x^{\star}, x(0)\right)>0 \\
\max \left(s^{\star}, s(0)\right) \geq s(t) \geq \min \left(s^{\star}, s(0)\right)>0
\end{array}\right.
$$

Using (H1b), we conclude that for any positive initial state conditions and for all non-negative time, the function $r($.$) and thus the manipulated variable u($.$) (follow-$ ing law (3)) is bounded below by a positive constant. Considering the closed loop system (4), it is straightforward to see that $\xi^{\star}$ is globally exponentially stable.

\subsection{Adaptive nonlinear controller design}

The controller proposed in section 3.1 requires perfect knowledge of the parameters $k / \lambda$ and $s_{i n}$ to perform the stabilization towards the targeted set point without static error. However, identification of these parameters is a difficult task, especially for bioprocesses. To solve this drawback, we propose an adaptive feedback control law based on a new information obtained from the plant:

Hypothesis $\mathbf{3}$ (H3): We assume that the state variable $s=y_{2}$ is available from the plant.

In the sequel, we suppose that $(\mathrm{H} 1),(\mathrm{H} 2)$ and $(\mathrm{H} 3)$ hold. Moreover $s^{\star}$, belongs to $\left(0, s_{i n}\right)$. Let us denote $\chi=$ $(x, s, p, \gamma)$ the new state vector and $\gamma^{\star}=k /\left(\lambda\left(s_{i n}-s^{\star}\right)\right)$.
Proposition 2: Under assumptions (H1), (H2) and (H3), the nonlinear adaptive feedback control law:

$$
\begin{aligned}
& \left\{\begin{array}{l}
u(.)=\gamma(t) y_{1}=\gamma(t) \lambda r(s, x, . .) \\
\dot{\gamma}=K y_{1}\left(s^{\star}-y_{2}\right)\left(\gamma-\gamma_{m}\right)\left(\gamma_{M}-\gamma\right)
\end{array}\right. \\
& \text { With: } 0<\frac{k}{\lambda s_{i n}}<\gamma_{m}<\gamma^{\star}<\gamma_{M} \text { and } K>0
\end{aligned}
$$

globally stabilizes system (2) towards the positive set point $\chi^{\star}=\left(x^{\star}, s^{\star}, p^{\star}, \gamma^{\star}\right)$.

Proof: Control law (6) yields to the closed loop system:

$$
\left\{\begin{array}{l}
\dot{x}=y_{1}\left(\frac{1}{\lambda}-\alpha \gamma x\right) \\
\dot{s}=y_{1}\left[\gamma\left(s_{i n}-s\right)-\frac{k}{\lambda}\right] \\
\dot{p}=y_{1}\left(\frac{h}{\lambda}-\beta \gamma p\right) \\
\dot{\gamma}=K y_{1}\left(s^{\star}-y_{2}\right)\left(\gamma-\gamma_{m}\right)\left(\gamma_{M}-\gamma\right)
\end{array}\right.
$$

In the sequel we will only consider positive initial conditions $x(0), s(0), p(0)$ and $\gamma(0)$ such that $\gamma(0) \in\left(\gamma_{m}, \gamma_{M}\right)$. With these initial conditions $x, s, p$ remain non-negative and $\gamma$ remains in $\left(\gamma_{m}, \gamma_{M}\right)$. Using $\gamma$ boundary values, as for equation (5), we show that $\forall t \geq 0$ the state variables $s(t)$ and $x(t)$ remain positive; thus using (H1b) we conclude that $y_{1}=\lambda r($.$) is bounded below by a posi-$ tive constant. We are now able to make the time change $t^{\prime}=\int_{0}^{t} y_{1}(\tau) d \tau$ (Chicone, 1999). Let us make the useful change of coordinate: $v=s_{i n}-s$. The closed loop system (7) becomes (denoting with a prime the time derivatives with respect to $t^{\prime}$, and $\left.v^{\star}=s_{i n}-s^{\star}\right)$ :

$$
\left\{\begin{array}{l}
x^{\prime}=\left(\frac{1}{\lambda}-\alpha \gamma x\right) \\
p^{\prime}=\left(\frac{h}{\lambda}-\beta \gamma p\right) \\
v^{\prime}=\gamma^{\star} v^{\star}-\gamma v \\
\gamma^{\prime}=K\left(v-v^{\star}\right)\left(\gamma-\gamma_{m}\right)\left(\gamma_{M}-\gamma\right)
\end{array}\right.
$$

The dynamical system (8) is an autonomous triangular system (Viel, Busvelle \& Gauthier, 1995): the system in $v$ and $\gamma$ does not depend upon the two other state variables $x$ and $p$. Now we consider the sub-system in $v$ and $\gamma$ :

$$
\left\{\begin{array}{l}
v^{\prime}=\gamma^{\star} v^{\star}-\gamma v \\
\gamma^{\prime}=K\left(v-v^{\star}\right)\left(\gamma-\gamma_{m}\right)\left(\gamma_{M}-\gamma\right)
\end{array}\right.
$$

First we want to show that the state of system (9) enters the set $\{v>0\}$ in finite time. Considering the dynamics of $v(t)$ in the set $v \leq 0$, we show that $v^{\prime} \geq \gamma^{\star} v^{\star}>0$, which proves that $v$ enters the set $\{v>0\}$ in finite time. In the sequel, we will consider the initial time (by time translation if necessary) belonging to the set $\mathcal{E}=\left\{v>0, \gamma \in\left(\gamma_{m}, \gamma_{M}\right)\right\}$. We introduce the Lasalle 
function used by Harrison (1979) in the context of Lyapunov stability for predator-prey models:

$W(v, \gamma)=\int_{v^{\star}}^{v} \frac{w-v^{\star}}{w} d w+\int_{\gamma^{\star}}^{\gamma} \frac{w-\gamma^{\star}}{K\left(w-\gamma_{m}\right)\left(\gamma_{M}-w\right)} d w$

We check that $W(v, \gamma)$ is defined, non-negative on $\mathcal{E}$ and vanishes only for $v=v^{\star}$ and $\gamma=\gamma^{\star}$. Furthermore:

$W^{\prime}=-\left(\frac{\gamma^{\star}}{v}\left(v^{\star}-v\right)^{2}\right)$

Thus, $W^{\prime}$ is defined and negative on $\mathcal{E}$ and vanishes only for $v=v^{\star}$. Lasalle's theorem implies that every solution of system (9) approaches the largest invariant set in the domain defined by $W^{\prime}=0$ (Khalil, 1992); let us denote this set $\Omega=\left\{v=v^{\star}, \gamma \in\left(\gamma_{m}, \gamma_{M}\right)\right\}$. Now, consider a trajectory initialized in $\Omega$ at $v=v^{\star}$ and $\gamma \neq \gamma^{\star}$; with respect to system (9) it is clear that this trajectory escapes from $\Omega$ and therefore that the largest invariant set in $\Omega$ is the fixed point $\left(v^{\star}, \gamma^{\star}\right)$. Then $\left(v^{\star}, \gamma^{\star}\right)$ is a globally attractive fixed point for system (9).

A straightforward Jacobian matrix computation at the point $\left(v^{\star}, \gamma^{\star}\right)$ proves that this fixed point is locally stable too. Then, we conclude that $\left(v^{\star}, \gamma^{\star}\right)$ is a globally asymptotically stable (GAS) fixed point for system (9).

Now let us study the behavior of the last two state variables $x$ and $p$ on the set defined by $v=v^{\star}$ and $\gamma=\gamma^{\star}$. The corresponding system is linear and has a single equilibrium $\left(x^{\star}, p^{\star}\right)=\left(\frac{1}{\lambda \alpha \gamma^{\star}}, \frac{h}{\lambda \beta \gamma^{\star}}\right)$ which is GAS. In order to finish the proof, we need the following result for autonomous triangular systems, which is proved by Viel et al. (2000).

Lemma: Consider the triangular system in $\mathbb{R}^{n}$ :

$(\Sigma)\left\{\begin{array}{l}\dot{z}=f(z, w) \\ \dot{w}=g(w)\end{array}\right.$

with: $z \in \mathbb{R}^{n-k}, w \in \mathbb{R}^{k}, f($.$) and g(.) \mathcal{C}^{1}$ functions. Moreover we assume that:

$A 1: w=0$ is a GAS fixed point for $\dot{w}=g(w)$

A2: $z=0$ is a GAS fixed point for $\dot{z}=f(z, 0)$

A3: every forward orbit of system $(\Sigma)$ is bounded

Then, 0 is a GAS fixed point for system $(\Sigma)$

Note that assumptions (A1) to (A3) hold for system (8). Thus applying the Lemma (after state translation) to system (8), we conclude that the fixed point defined by $v=v^{\star}, \gamma=\gamma^{\star}, x=x^{\star}$ and $p=p^{\star}$ is a GAS fixed point for (8). Thus, coming back to the original time and state variables, we conclude that the control law (6) globally stabilizes system (2) towards the point $\chi^{\star}$.
Remark: If we want to regulate $x$, we can build an adaptive control using $x$ measurements with a set point $x^{\star}$. For example for $x$ we have:

$\dot{\gamma}=K\left(x-x^{\star}\right)\left(\gamma_{m}-\gamma\right)\left(\gamma_{M}-\gamma\right)$

with: $0<\frac{k}{\lambda s_{i n}}<\gamma_{m}<\frac{1}{\lambda \alpha x^{\star}}<\gamma_{M}$

The same kind of adaptation can be done for p regulation.

Remark: Suppose kinetics measurement is corrupted by a (small) relative perturbation $\eta(t)$ such that $y_{1}=(1+$ $\eta) r($.$) . Then, since y_{1}$ is in factor in system (4) (resp. (7)), one can show the state remains asymptotically in a ball centered on $\xi^{\star}\left(\chi^{\star}\right)$, of a radius proportional to $\eta$ amplitude, highlighting controller's robustness.

\subsection{The problem of discrete time $y_{2}$ measurements}

In practice the substrate $s$ is sometimes only available at low frequency time measurements $y_{2}(i T)=s(i T)(T$ the sampling period). We show that the control law (6) still works, but requires slow adaptation, i.e. a small $K$.

Indeed, we can choose a $K$ small enough ensuring that $\gamma$ is a slow variable of the closed loop system while $s, x, p$ are fast ones. Singular perturbation theory (Khalil, 1992) applies, such that $s$ remains on the manifold $s=s_{i n}-k / \lambda \gamma$. Straightforward calculus shows that the adaptive equation is such that $\forall t \in[i T,(i+1) T)$ :

$\dot{\gamma}=y_{1} \frac{K k}{\lambda \gamma^{\star} \gamma(i T)}\left(\gamma^{\star}-\gamma(i T)\right)\left(\gamma-\gamma_{m}\right)\left(\gamma_{M}-\gamma\right)$

Integrating this equation between $i T$ and $(i+1) T$, we find the recurrent expression of $\gamma((i+1) T)$. Then, we show that a sufficient condition for the convergence of the sequence $(\gamma(i T))_{i \in \mathbb{N}}$ towards $\gamma^{\star}$ is:

$0<T<\frac{1}{K}\left[\frac{2 \lambda \gamma^{\star} \gamma_{m}}{k \max \left(y_{1}\right)\left(\gamma_{M}-\gamma_{m}\right)^{2}}\right]$

Then for all sampling period $T$, there exists a small enough $K$, such that $T$ fulfills this necessary condition.

\section{Real life example: an anaerobic WWTP}

Anaerobic digestion (AD) consists of a succession of biological degradations that take place in the absence of oxygen and by which organic matter is decomposed and bioconverted on the one hand into biogas (i.e., a mixture of $\mathrm{CO}_{2}$ and $\mathrm{CH}_{4}$ ) and, on the other hand, into microbial biomass and residual organic matter.

The main advantages of AD processes for water treatment are a good capacity to treat substrates at high concentrations, and the possibility for energy recovery 
through methane combustion. However, many industrials are still reluctant to use AD processes, because of an intrinsic disadvantage: they have two stable equilibrium points (Bernard et al., 2001). One is an operating point while the other corresponds to biomass washout. $\mathrm{AD}$ processes require therefore a regulation procedure to work safely. Moreover, the control's robustness towards kinetics modelling is important, since there does not exist any reliable model.

We thus propose a very simple model, describing the key features of AD, derived from (Bailey \& Ollis, 1986). This model focuses only on the main bioreaction and is thus a basic summary of the whole bioreaction. Denoting $S$ the chemical oxygen demand (COD, pollutant matter), $X$ the bacterial population performing anaerobic digestion to produce methane, we have the reaction (1), and:

$r(s, x, .)=.\mu(s) x$

The specific growth rate $\mu($.$) of biomass X$ is often represented as a Haldane law:

$\mu(s)=\frac{\mu_{m} s}{K_{m}+s+s^{2} / K_{i}}$

with $\mu_{m}, K_{m}, K_{i}$ biological (positive) constants. Note that with this expression for $r(s, x)$, hypothesis (H1) is fulfilled. The model for compounds concentration is of the form (2), but with no equation for the produced methane. Indeed, since the methane solubility is very low, the produced methane is mainly gaseous. Then dissolved methane remains at steady state and of zero concentration. Moreover, the gaseous outflow of methane can be easily measured on the plant, so that (H2) holds:

$Q_{C H_{4}}=\lambda r(s, x)=y_{1}$

\subsection{Simulations}

AD's model is simulated to evaluate the benefits of the control law (6) through comparison between open loop and closed loop performances. Together with a substrate concentration set point $s^{\star}=4 \mathrm{gCOD} / \mathrm{L}$, we assume the following realistic parameters' values $\alpha=0.8, \mu_{m}=0.9$ day $^{-1}, K_{m}=9 \mathrm{~g} / \mathrm{L}, K_{i}=3 \mathrm{~g} / \mathrm{L}, k=2, \gamma_{m}=0.1334$ $\mathrm{L} / \mathrm{g}, \gamma_{M}=0.4 \mathrm{~L} / \mathrm{g}$ and $K=0.8$. Results are shown in Figure 1. For the open loop model, it results in applying the dilution $u^{\star}=\mu\left(s^{\star}\right) / \alpha=0.2455$ day $^{-1}$, while for the closed loop model, the control law is computed from the expression (6). We choose a piecewise constant influent pollutant concentration $s_{i n}$ (unknown) to highlight the adaptive controller's action. It must be kept in mind that the destabilization (see e.g. the open loop system in Figure 1) of the process implies the disappearance of the biomass from the digester. Then the digester has to be inoculated again, which lasts for months, while wastewater is no longer treated.
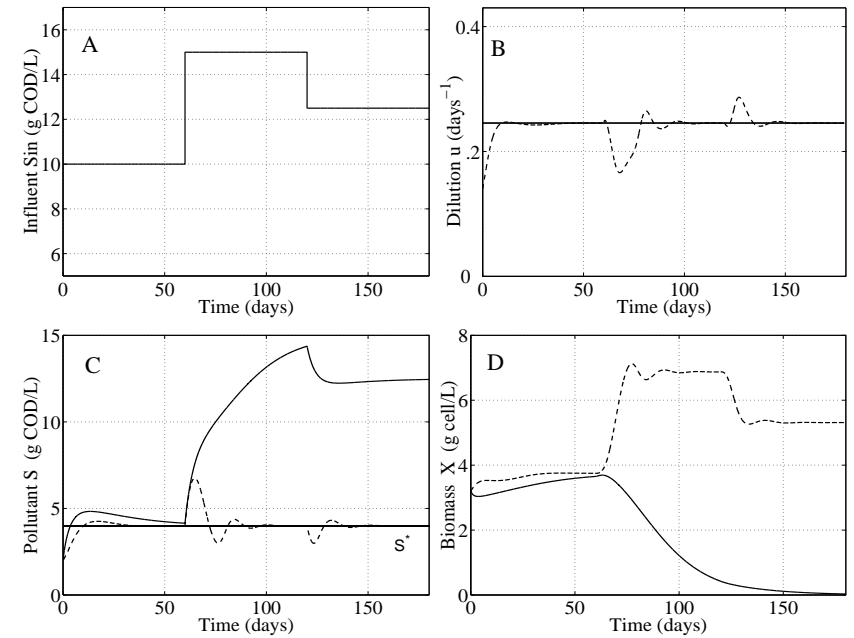

Fig. 1. Simulations of the open loop plant (continuous line) and of the closed loop plant with the control law (6) (dashed line) (figures B, C, D). On figure A the piecewise constant influent concentration $s_{i n}$ (continuous line) is shown. Note that the step in $s_{i n}$ is lethal for the open loop digester.

Indeed from $t=0$ to $t=60$ both the open loop and the closed loop systems converge towards the equilibrium corresponding to the set point $s=s^{\star}$. At $t=60$, influent substrate concentration increases from 10 to $15 \mathrm{gCOD} / \mathrm{L}$. On the one hand, it results in the destabilization of the open loop process: the biomass starts to be washed out of the bioreactor. On the other hand, the closed loop process escapes from the equilibrium $s=s^{\star}$ for a short time but, the control law (6) drives the state variables back towards the equilibrium corresponding to the set point $s=s^{\star}$; the change of $s_{i n}$ has been efficiently rejected. Then, at $t=120, s_{\text {in }}$ decreases from 15 to $12.5 \mathrm{gCOD} / \mathrm{L}$. Destabilization of the open loop system still goes on though the decrease of $s_{i n}$. The control law remains efficient and rejects the $s_{i n}$ change again.

\subsection{Real Life Experiments}

An experimental test of the controller has been performed on a fully instrumented fixed bed anaerobic digester (Steyer, Bouvier, Conte, Gras \& Sousbie, 2002), located in Narbonne (France), at the "Laboratoire de Biotechnologie de l'Environnement" (LBE) of INRA. Raw industrial distillery wastewaters obtained from local wineries in the area of Narbonne, France, were used. They have different characteristics according to the wineries where the wastewater is taken from. The process is a pilot-scale up-flow anaerobic fixed bed reactor and has a useful volume of about $1 \mathrm{~m}^{3}$ (Steyer et al., 2003).

Two transient changes obtained for the input variable and for the controlled variable $s$ using the control law (3) are presented in Figure 2. Transients are presented for two different set points values $s^{\star}$. These experiments 
perfectly agree with the expected qualitative behavior of the closed loop plant (a first order with an unsettled gain) and thus validate our approach on a real life process. Unfortunately, the adaptive version of the control law (6) has not been tested yet.
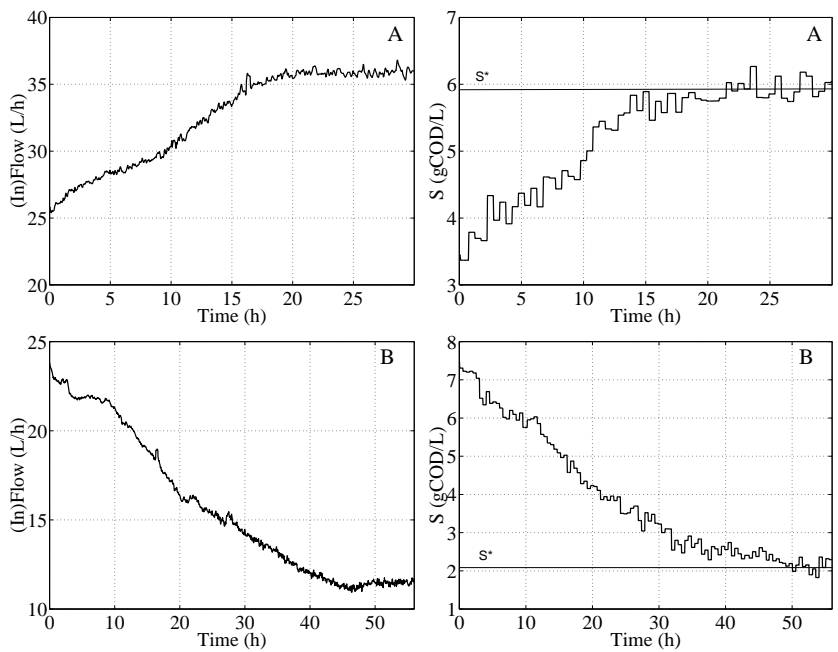

Fig. 2. Input (inflow) and $s$ behaviors during two transients: cases $\mathrm{A}$ and $\mathrm{B}$

Nevertheless, the practical interest of the control law was also demonstrated in an additional experiment (see Figure 3). In this case, a technical problem in the feeding line indeed lead to feed the process with pure water (instead of vinasses) while the control law was driving the process to a new steady state. This experiment is as well representative of the presence of a toxicant in the feeding (Mailleret et al., 2003).
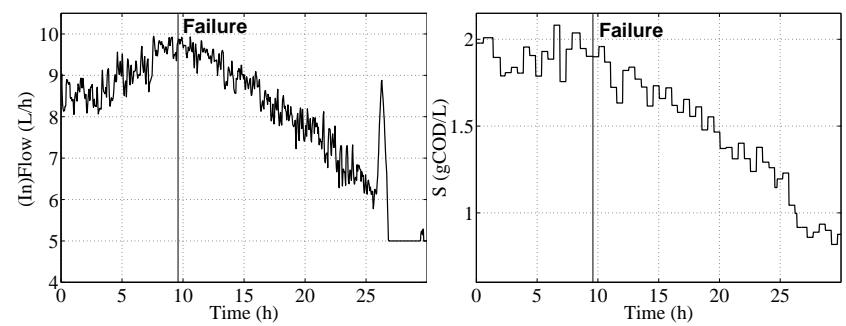

Fig. 3. Input (inflow) and $s$ behaviors as the digester is fed with water

This failure happens at $t=9 h$ in Figure 3 . Reaction of the control law was then particularly safe since it decreased the feed flow down to its minimal technological value. It is to be mentioned that most other control laws (e.g. PID controllers) would have instead increased the feed flow to maintain the COD concentration at the desired value. In such a case, washout of the bacterial population would probably occur. This safety characteristic of our control law is a key advantage for practical applications and allows us to foresee industrial implementation.

\section{Conclusion}

We have proposed two control laws for the regulation of a generic model of simple bioreactions in continuous stirred tank bioreactors. We have proved the global asymptotic stability of both closed loop systems towards a chosen operating point, while the laws fulfill the input non-negativity constraint. Moreover, no assumption has been made about the analytical expression of the bioreaction's kinetics $r($.$) . In addition, these control laws are$ robust to a relative noise on $r($.$) measurements and the$ adaptive law does not require any model parameters.

Some other work shows that the controllers can also be applied to a broader class of positive systems (Mailleret, 2003), and to more complex bioreaction schemes such as variable yield bioreactions (Mailleret, Gouzé \& Bernard, 2003) or "cascade" bioreactions (Mailleret et al., 2003).

Finally, the real-life experiments we performed on a pilot-scale anaerobic WWTP show the practical relevance of our approach, together with an unexpected safe behavior towards some process failures.

Acknowledgment: This research has been supported by the European project IST-2000-28156 TELEMAC.

\section{References}

Bailey, J.E. \& Ollis, D.F. (1986). Biochemical engineering fundamentals (2nd ed.). McGraw-Hill chemical engineering series.

Bastin, G. \& Dochain, D. (1990). On-line estimation and adaptive control of bioreactors. Elsevier.

Bastin, G. \& Van Impe, J.F. (1995). Nonlinear and adaptive control in biotechnology: a tutorial. European Journal of Control, 1, 37-53.

Bernard, O., Hadj-Sadok, Z., Dochain, D., Genovesi, A. \& Steyer, J-P. (2001). Dynamical model development and parameter identification for an anaerobic wastewater treatment process. Biotech. Bioeng., 75, 424-438.

Chicone, C. (1999). Ordinary differential equations with applications. Springer.

Farza, M., Busawon, K. \& Hammouri, H. (1998). Simple nonlinear observers for on-line estimation of kinetic rates in bioreactors. Automatica, 34, 301-318.

Gouzé, J-L., Rapaport, A. \& Hadj-Sadok, Z. (2000). Interval observers for uncertain biological systems. Ecological modelling, 133, 45-56.

Harrison, G.W. (1979). Global stability of predator-prey interactions. J. of Mathematical Biology, 8, 159-171.

Heinzle, E., Dunn, I.J. \& Ryhiner, G.B. (1993). Modeling and control for anaerobic wastewater treatment. Advances in Biochemical Engineering and Biotechnology, 48, 79-114.

Khalil, H. K. (1992). Nonlinear systems. Macmillan Publishing Company. 
Mailleret, L., Bernard, O. \& Steyer, J-P. (2003). Robust regulation of anaerobic digestion processes. $\mathrm{Wa}$ ter Science and Technology, 48, 87-94.

Mailleret, L., Gouzé, J-L. \& Bernard, O. (2003). Nonlinear control for algae growth models in the chemostat. Proceedings of the European Control Conference (ECC03), Cambridge, U.K.

Mailleret, L. (2003). Positive Control for a Class of Nonlinear Positive Systems. In L. Benvenuti, A. De Santis \& L. Farina (Eds.), Positive Systems, Lecture Notes in Control and Information Sciences, Vol. 294 (pp. 175182). Springer.

Perrier, M. \& Dochain, D. (1993). Evaluation of control strategies for anaerobic digestion processes. International Journal of Adaptive Control and Signal Processing, 7, 309-321.

Proell, T. \& Karim, N.M. (1994). Nonlinear control of a bioreactor using exact and $\mathrm{I} / \mathrm{O}$ linearization. International Journal of Control, 60, 499-519.

Rapaport, A. \& Harmand, J. (2002). Robust regulation of a class of partially observed nonlinear continuous bioreactors. Journal of Process Control, 12, 291-302.

Steyer, J-P., Bouvier, J-C., Conte, T., Gras, P. \& Sousbie, P. (2002). Evaluation of a four year experience with a fully instrumented anaerobic digestion process. Water Science and Technology, 45, 495-502.

Viel, F., Busvelle, E. \& Gauthier, J-P. (1995). Stability of polymerization reactors using I/O linearization and a high-gain observer. Automatica, 31, 971-984. 